\title{
FLUID OPTIMIZATION WITH HYDROXYETHYL STARCH 130/0.4 COMPARED WITH MODIFIED FLUID GELATIN GUIDED BY ESOPH- AGEAL DOPPLER DURING MAJOR ABDOMINAL SURGERIES

\author{
By
}

GEHAN G. EL-FANDY ${ }^{1}$, SOHAILA H. OMAR ${ }^{1}$, AYMAN A. EL-DESOUKY ${ }^{2}$, HANAN F. KHAFAGY ${ }^{1}$, MOHAMED M. HUSSIEN ${ }^{1}$, HEND H. KAMEL ${ }^{1}$, AHMED I. REFAAT ${ }^{1}$.

Department of Anesthesiology ${ }^{1}$, Theodor Bilharz Research Institute, Ministry of Scientific Research, Imbaba P.O. 30, Giza, and Department of Anesthesiology ${ }^{2}$, Faculty of Medicine, Cairo University, Ministry of Higher Education, Egypt.

\begin{abstract}
Perioperative fluid therapy has a direct bearing on patient outcome and accordingly should be tailored individually. Thus the efficacy of HES 130/0.4 was compared to modified fluid gelatin for volume expansion during major abdominal surgery guided by transesophageal Doppler (TED). Fifty adult patients ASA physical status I-II undergoing major abdominal surgery were anesthetized with standard technique. In addition to basal fluid requirement, patients were randomly allocated into two equal groups (25 patients each) to receive $200 \mathrm{cc}$ of either 6\% HES 130/0.4 (HES group) or 3\% modified fluid gel (GEL group) as intraoperative colloid replacement guided by TED. Heart rate, mean arterial blood pressure, central venous pressure, and Doppler derived measurements were recorded at the following timings: T1; after induction, T2; after skin incision, T3; two hours after that and T4; at the end of surgery. Fluid administration and transfusion requirements were recorded. Laboratory tests for hemostasis, hepatic and renal functions were continued till the fifth postoperative day. Both groups were comparable regarding Doppler derived data and fluid balance. Platelet count showed a significant drop $(p<0.05)$ in group GEL in all postoperative days compared with baseline and with the group HES. Prothrombin time and INR showed a significant increase while prothrombin concentration showed a significant drop, throughout 5 postoperative days in group HES while in the 3 postoperative days in group GEL $(p<0.05)$ but comparable between groups. Both groups showed postoperative drop in creatinine level and postoperative rise of liver function tests. HES 130/0.4 and modified gelatin have comparable fluid optimization effect guided by TED in major abdominal surgeries. HES 130/0.4 has a more favorable effect on platelet counts than modified gelatin.

Key words: Abdominal surgeries, Esophageal Doppler, Fluid optimization, Gelatin, HES $130 / 0.4$.
\end{abstract}

\section{Introduction}

Plasma volume expansion is substantial importance during major surgery and optimizing preload and cardiac output in these patients reduces postoperative complications and length of hospital stay. The dilemma is which type of fluid can be administered (colloid versus crystalloid), total volume administered (liberal versus restrictive), and the goal needed to guide fluid administration (goal directed versus traditional parameters). The optimal strategy for fluid therapy re- mains controversial and uncertain (Chappell et al, 2008).

Colloids, being more effective in increasing blood volume and cardiac output, may be preferred to crystalloids during major surgery. The effect of fluid replacement on the intravascular volume, coagulation cascade, microcirculation, and any possible allergic reactions must be considered (Van Der Linden et al, 2013). Administration of synthetic plasma expanders protects against adverse events of allogeneic blood (Mastroianni et $a l$, 1994). Gelatins [GEL] and hydroxyethyl 
starches [HESs] are synthetic colloids which vary significantly in their chemical, pharmacokinetic, and pharmacodynamic properties. All synthetic colloids have dose-related side effects involving; coagulopathy, renal failure, and tissue storage (Van Der Linden et al, 2013).

Gelatins are polypeptides that have the advantage of their unlimited daily dose recommendation and minimal effect on hemostasis. However, they are associated with a more frequent incidence of allergic reactions (Van Der Linden et al, 1992). HESs are modified natural polysaccharides that have the advantage of higher plasma expanding effect and an infrequent incidence of allergic reactions, but more pronounced effects on hemostasis than GEL (Treib et al, 1992). Voluven ${ }^{\circledR}$ is a $6 \%$ HES $(130 / 0.4)$ solution in $0.9 \%$ sodium chloride which appears to have fewer hemostatic effects due to its lower molecular weight (Jungheinrich et al, 2004). Fluid resuscitation with HES 130/0.38-0.45 may reduce the risk of bleeding associated with synthetic colloids of higher molecular weight and degree of substitution (Franz et $a l$, 2001). On the other hand, it was found that HES induces delayed initiation of sufficient thrombin generation and impaired platelet function, and GEL induces derangements of fibrin polymerization. These results may be induced not only from hemodilution but also due to their pharmacological properties (Jin and $\mathrm{Yu}, 2010$ ). Biochemical data representing liver and kidney function remained within normal limit after the off-pump coronary artery bypass surgery when using HES (Kim et al. 2007). However, in meta-analysis conducted in 2012 concluded that the poor quality and small size of available studies don't allow the benefits and risks of HES 130/ 0.4 to be reliably estimated (Gattas et al, 2012).

As anesthetists target hemodynamic optimization and adequate perioperative organ perfusion, they strive for maintenance of balance between effective circulatory volume and avoidance of fluid overload or re- striction (with resultant occult hypovolemia). Both of them cannot be detected by standard hemodynamic monitoring and remains unchanged via normal homeostatic mechanisms (Mythen and Webb, 1994). Adequate perfusion, however, not only relies on sufficient perfusion pressure but also on systemic blood flow, i.e. cardiac output (CO) which is not routinely monitored and may require the use of invasive pulmonary artery catheter. The development of Transesophageal Doppler (TED) ultrasonography of the descending aorta could be a useful, minimally invasive monitoring device. It allows a continuous estimation of $\mathrm{CO}$ and facilitates the assessment of preload, afterload, and myocardial contractility by calculating advanced hemodynamic variables (Dark and Singer, 2004). TED monitoring is more reliable tool to monitor goal directed therapy (GDT) for titrating fluid and optimizing preload over the traditional hemodynamic parameters during major surgery (Gan et al, 2002).

Thus this study was designed to evaluate intravascular optimization with hydroxyethyl starch 130/0.4 compared to modified fluid gelatin, guided by esophageal Doppler during major abdominal surgeries. The hypothesis of this study is that the HES (130/0.4) may be more effective than GEL in restoring the hemodynamics. The primary outcome of the study was estimating the cardiac index (CI) values in both types of colloids. The secondary outcomes were the other TED parameters, conventional hemodynamic parameters, liver and kidney functions as well as coagulation profile in both groups.

\section{Subjects, Materials and Methods}

After ethical committee approval and patients' informed consents, the study was conducted at Theodor Bilharz Research Institute on 50 ASA physical statuses I, II adult patients scheduled for major abdominal surgery as (e.g. cancer bladder, colon, stomach or pancreas). Patients were randomly allocated to receive in addition to their standard basal requirements of fluid 
management, either 6\% HES 130/0.4 (Voluven $^{\circledR}$, Fresenius Kapi, Germany) (group HES: $n=25$ ) or $3 \%$ modified fluid Gelatin (Hemagel, Vacsera, Egypt) (group GEL: $n=25$ ) as their colloid during the intraoperative period, with a maximum dosage of $50 \mathrm{ml} / \mathrm{kg}$. Randomization was done by the attending resident opening a sealed envelope. Patients who had medical histories of recent myocardial infarction, congestive heart failure (left ventricular ejection fraction $<40 \%$ ), esophageal pathology, anemia (hemoglobin $<10 \mathrm{~g} / \mathrm{dl}$ ), renal insufficiency (serum creatinine $>1.2 \mathrm{mg} / \mathrm{dl}$ ), liver dysfunction (aspartate aminotrans-ferase (AST) or alanine aminotransferase (ALT] $>40$ U/L), uncontrolled diabetes mellitus, chronic obstructive pulmonary disease and/or an allergy to HES or GEL were excluded from this study. Patients who had coagulopathy (platelet count $<100 \times 10^{9} / \mathrm{L}$, prothrombin time (PT] $>16 \mathrm{~s}$ and INR $>1.2$ ) and/or medication with drugs known to affect blood coagulation or patient required intraoperative massive blood transfusion were also excluded.

Patients were premedicated with midazolam $0.05 \mathrm{mg} / \mathrm{kg}$ given intravenously half an hour before induction of anesthesia. Ringer's acetate solution was infused as a preload $(500 \mathrm{ml})$ then continued at a rate of $6-8$ $\mathrm{ml} / \mathrm{kg} / \mathrm{h}$ during surgery for supplying maintenance and deficit. The following monitors were attached to the patients: five leads ECG, non invasive blood pressure, $\mathrm{SpO}_{2}$, capnography, anesthetic gas analyzer, temperature and peripheral nerve stimulator (Infinity Kappa, Dräger, Lübeck, Germany). Anesthesia was induced with IV fentanyl 1$2 \mu \mathrm{g} / \mathrm{kg}$ and IV propofol $1.5-2 \mathrm{mg} / \mathrm{kg}$ until loss of verbal contact. Neuromuscular blockade was achieved by IV atracurium 0.5 $\mathrm{mg} / \mathrm{kg}$ followed by tracheal intubation. Anesthesia was then maintained using isoflurane to keep the end-tidal anesthetic concentrations within $0.8-1.2 \%$ with fresh gas flow oxygen in air $30-40 \%$ and mechanical ventilation was adjusted to maintain $\mathrm{PaCO}_{2}$ be- tween 30 and $35 \mathrm{~mm} \mathrm{Hg}$. The esophageal Doppler (CardioQ, Deltex Medical, Chichester, UK) probe was then inserted orally and positioned approximately $35-40 \mathrm{~cm}$ from the teeth. TED measures the velocity of blood flow in the descending thoracic aorta. Integrating the velocity-time curve gives the distance traveled by the blood following cardiac systole and multiplying this by the cross-sectional area (estimated by a nomogram) derives stroke volume and cardiac output.

Patient demographic data, standard hemodynamic data including heart rate (HR), mean arterial blood pressure (MAP), central venous pressure (CVP), and urine output were registered. Doppler derived data including cardiac index (CI), stroke volume (SV), stroke volume index (SVI), peak velocity, systolic flow time, oxygen delivery index $\left(\mathrm{DO}_{2} \mathrm{I}\right)$ and central venous oxygen saturation $\left(\mathrm{SCVO}_{2}\right)$, were also recorded. Readings were taken after induction of anesthesia before skin incision T1, after laparotomy T2, 2 hours after skin incision T3, and at the end of surgery T4. In addition to the standard fluid replacement, $200 \mathrm{cc}$ of $6 \%$ HES $130 / 0.4$ or $3 \%$ modified fluid Gelatin were given to a maximum volume of $50 \mathrm{ml} / \mathrm{kg}$ targeting corrected flow time (FTc) $>0.35$ seconds (a trace obtained from the TED). The FTc is the systolic flow time corrected for HR and it is considered as a sensitive cardiac-filling indicator. The patients were guided by a dynamic TED fluid protocol as follows: Fluid challenge by colloid (200 cc) was infused when FTc was less than 0.35 second. If the stroke volume was maintained or increased and the FTc remain below 0.35 second, the fluid challenge was repeated. If the stroke volume rose by more than $10 \%$ but the FTc exceeded 0.35 second, the fluid challenge was repeated until no further rise in stroke volume occurred. If the FTc rose above 0.40 second with no change in stroke volume, indicating that intravascular volume was optimized, further fluid was not admin- 
istered until the FTc or stroke volume fell by $10 \%$ (Gan et al, 2002).

Blood transfusion was given when hemoglobin level (less than $7 \mathrm{~g} / \mathrm{d}$ ), platelet transfusion was given if platelet count $<50.000 / \mathrm{dl}$ and fresh-frozen plasma was given if INR $>1.5$ or abnormal bleeding in the surgical field. Blood losses, intravenous fluid administration, volume of allogenic blood transfusion as well as number of patients needed platelets or fresh frozen plasma (FFP) transfusion were recorded.

At the end of surgery, neuromuscular blockade will be antagonized with 0.05 $\mathrm{mg} / \mathrm{kg}$ neostigmine and $0.02 \mathrm{mg} / \mathrm{kg}$ atropine. Postoperative analgesia was provided by intravenous infusion of $1 \mathrm{gm}$ acetaminophen and IV mepridine $1 \mathrm{mg} / \mathrm{kg}$ every 12 hours. Laboratory Measurement including hemoglobin concentration $(\mathrm{Hb})$, hematocrite (Hct), platelet count (PLT), prothrombin time (PT), prothrombin concentration (PC) International Normalized Ratio (INR), serum creatinine $(\mathrm{Cr})$, ALT, AST, total bilirubin were recorded preoperative then every day for 5 days after surgery.

Statistical analysis: Estimation of the sample size was performed using program $G$ Power 3.1 (http://www.psycho.uni-duesseldorf.de). For an effect size of 0.20 , assuming a two-sided type I error of 0.05 and a power of 0.80 , a sample size of 50 patients ( 25 patients in each group) would be required. Results are expressed as mean \pm standard deviation or number (\%). Comparison between the two groups was performed using Mann Whitney U test. Comparison relative to the baseline in the same group was performed by Friedman's ANOVA with post hoc Wilcoxon matched pairs. Categorical data were compared by Chi-square test. SPSS computer program (version 12 windows) was used for data analysis. P value $\leq 0.05$ was considered significant.

\section{Results}

All patients enrolled completed the study period; their demographic data and duration of anesthesia were comparable between both groups (Tab.1).

Standard hemodynamic data (HR and MAP) showed increased different readings (T2, T3 and T4) in both groups when compared to $\mathrm{T} 1$ and significant higher values in group GEL than group HES at T3 $(P<0.05)$. CVP was significantly higher in group GEL than group HES at T2 and T3 $(P<0.05)$. Doppler derived data (CI, SV, SVI, peak velocity and systolic flow time), showed elevations in their values at different readings in both groups when compared to T1 level. $\mathrm{DO}_{2} \mathrm{I}$ was significantly increased $(P<0.05)$ at $\mathrm{T} 2$ in group HES and at T2, T3 and T4 in group GEL when compare to T1 level. The percent of $\mathrm{SCVO}_{2}$ showed significant rise $(P<0.05)$ at $\mathrm{T} 2$ in both group and significant drop $(P<0.05)$ at T4 in group HES when compared to T1 level (Tab. 2).

As for blood loss and replacement, the total volume of crystalloid and colloid administered, number of patients required plasma and platelet transfusion as well as urine output, although higher in group HES than group GEL yet was statistically insignificant (Tab. 3).

$\mathrm{Hb}$ and Hct levels were significantly lower $(P<0.05)$ in days $1-5$ postoperatively compared with baseline in both groups. No significant difference was found between the two groups except in the $5^{\text {th }}$ postoperative day where Hct level was significantly lower $(P<0.05)$ in group GEL than group HES. Platelet counts showed a postoperative drop in group HES on $2^{\text {nd }}$ postoperative day; while in group GEL there was a significant drop $(P<0.05)$ of their counts in all postoperative days compared with the preoperative reading and compared with the group HES. Coagulation tests showed significant prolongation of PT, significant reduction in PC and significant increase in INR in all postoperative days in group HES and in the first three days in group GEL compared with the baseline $(P<0.05)$, yet remained comparable between both groups (Tab. 4). 
Serum creatinine level revealed a significant decrease $(P<0.05)$ from day 3 to day 5 in group HES and from day 4 to day 5 in group GEL compared to preoperative values. Data was comparable between both groups in the five days following surgery except on $2^{\text {nd }}$ postoperative day where it was significantly lower $(P<0.05)$ in group HES than group GEL. As regards liver functions; serum AST levels showed a significant increase $(P<0.05)$ in first three postoperative days in both groups which extended to the fourth day in group GEL compared with the preoperative value. AST level was significantly higher $(P<0.05)$ in group HES than group GEL only on the first postoperative day. Serum ALT levels showed no significant differences within group HES or between groups, while in group GEL there was a significant increase $(P<0.05)$ in day 1 and 2 compared to baseline. There was a significant rise $(P<0.05)$ in bilirubin level postoperatively in day 1 in group HES and in day 2 and 3 in group GEL compared to baseline (Tab. 5).

Table 1: Demographic features and duration of surgery

\begin{tabular}{|l|c|c|}
\hline Items & HES group $(\mathrm{n}=25)$ & GEL group $(\mathrm{n}=25)$ \\
\hline Age $(\mathrm{yr})$ & $52.68 \pm 12.74$ & $59.00 \pm 13.52$ \\
\hline Gender $(\mathrm{M} / \mathrm{F})$ & $20 / 5(80 / 20 \%)$ & $20 / 5(80 / 20 \%)$ \\
\hline ASA class $(\mathrm{I} / \mathrm{II})$ & $12 / 13(48 / 52 \%)$ & $13 / 12(52 / 48 \%)$ \\
\hline Weight $(\mathrm{Kg})$ & $66.64 \pm 7.63$ & $65.32 \pm 13.07$ \\
\hline Height $(\mathrm{cm})$ & $166.84 \pm 4.34$ & $166.80 \pm 5.22$ \\
\hline Anesthetic duration $(\mathrm{h})$ & $5.77 \pm 0.94$ & $5.54 \pm 1.09$ \\
\hline
\end{tabular}

Data are expressed as mean \pm SD or number (\%).

Table 2: Hemodynamic data

\begin{tabular}{|c|c|c|c|c|c|}
\hline & Groups & $\mathrm{T} 1$ & $\mathrm{~T} 2$ & $\mathrm{~T} 3$ & $\mathrm{~T} 4$ \\
\hline \multirow[t]{2}{*}{ HR (beats/min) } & HES group & $77.60 \pm 2.25$ & $83.76 \pm 9.39^{b}$ & $78.68 \pm 10.75$ & $84.56 \pm 11.05^{b}$ \\
\hline & GEL group & $74.08 \pm 9.45$ & $85.20 \pm 14.58^{b}$ & $86.64 \pm 11.76^{b c}$ & $87.28 \pm 8.27^{b}$ \\
\hline \multirow[t]{2}{*}{ MAP (mm Hg) } & HES group & $91.88 \pm 18.34$ & $104.76 \pm 16.09^{b}$ & $92.64 \pm 9.72$ & $94.36 \pm 10.72$ \\
\hline & GEL group & $85.28 \pm 7.57$ & $109.72 \pm 9.51^{b}$ & $98.72 \pm 8.77^{b d}$ & $93.32 \pm 11.21^{\mathrm{a}}$ \\
\hline \multirow[t]{2}{*}{ CVP (mmHg) } & HES group & $5.68 \pm 2.85$ & $5.72 \pm 2.56$ & $6.00 \pm 1.66$ & $6.52 \pm 1.16$ \\
\hline & GEL group & $7.04 \pm 3.19$ & $7.80 \pm 2.38^{\mathrm{ad}}$ & $7.88 \pm 1.36^{\mathrm{d}}$ & $7.08 \pm 1.47$ \\
\hline \multirow[t]{2}{*}{$\mathrm{CI}\left(\mathrm{L} / \mathrm{min} / \mathrm{m}^{2}\right)$} & HES group & $3.40 \pm 1.10$ & $3.85 \pm 1.15^{b}$ & $3.89 \pm 1.57^{\mathrm{a}}$ & $4.34 \pm 1.25^{b}$ \\
\hline & GEL group & $2.92 \pm 1.08$ & $3.60 \pm 1.02^{b}$ & $3.89 \pm 0.98^{b}$ & $4.06 \pm 1.22^{b}$ \\
\hline \multirow[t]{2}{*}{$\mathrm{SV}(\mathrm{ml})$} & HES group & $72.40 \pm 22.16$ & $74.60 \pm 27.57$ & $82.16 \pm 25.42^{a}$ & $87.32 \pm 17.78^{b}$ \\
\hline & GEL group & $68.04 \pm 22.90$ & $76.76 \pm 16.83^{b}$ & $80.28 \pm 23.49^{b}$ & $79.12 \pm 23.75^{b}$ \\
\hline \multirow[t]{2}{*}{ SVI $\left(\mathrm{ml} / \mathrm{m}^{2}\right)$} & HES group & $42.62 \pm 13.82$ & $44.18 \pm 17.32$ & $49.16 \pm 18.13^{\mathrm{a}}$ & $51.66 \pm 13.14^{b}$ \\
\hline & GEL group & $38.12 \pm 11.82$ & $43.36 \pm 9.52^{b}$ & $45.32 \pm 11.02^{b}$ & $45.56 \pm 10.45^{b}$ \\
\hline \multirow{2}{*}{$\begin{array}{l}\text { Peak velocity } \\
(\mathrm{cm} / \mathrm{sec})\end{array}$} & HES group & $62.96 \pm 24.14$ & $71.16 \pm 25.29^{b}$ & $74.64 \pm 32.08^{b}$ & $75.40 \pm 25.15^{b}$ \\
\hline & GEL group & $59.56 \pm 24.43$ & $63.40 \pm 26.61^{\mathrm{a}}$ & $73.60 \pm 31.21^{b}$ & $74.12 \pm 33.17^{b}$ \\
\hline \multirow{2}{*}{$\begin{array}{l}\text { Systolic flow } \\
\text { time (msec) }\end{array}$} & HES group & $366.08 \pm 32.78$ & $385.36 \pm 55.44$ & $406.72 \pm 23.71^{b}$ & $414.92 \pm 25.28^{b}$ \\
\hline & GEL group & $357.52 \pm 38.66$ & $412.20 \pm 37.29^{b}$ & $403.52 \pm 19.39^{b}$ & $408.80 \pm 13.17^{b}$ \\
\hline \multirow{2}{*}{$\begin{array}{l}\mathrm{DO}_{2} \mathrm{I} \\
\left(\mathrm{cc} / \mathrm{min} / \mathrm{m}^{2}\right)\end{array}$} & HES group & $527.44 \pm 178.21$ & $562.92 \pm 169.92^{b}$ & $496.16 \pm 194.43$ & $515.44 \pm 143.19$ \\
\hline & GEL group & $445.56 \pm 165.56$ & $508.3 \pm 164.79^{b}$ & $522.08 \pm 184.80^{b}$ & $521.96 \pm 204.73^{b}$ \\
\hline \multirow[t]{2}{*}{$\mathrm{SCVO}_{2}(\%)$} & HES group & $90.56 \pm 4.81$ & $93.36 \pm 4.25^{b}$ & $89.40 \pm 6.34$ & $88.40 \pm 6.31^{b}$ \\
\hline & GEL group & $88.08 \pm 5.4$ & $91.12 \pm 4.87^{\mathrm{a}}$ & $88.40 \pm 6.03$ & $87.96 \pm 7.67$ \\
\hline
\end{tabular}

Data are expressed as mean \pm SD. T1; after induction, T2; after skin incision, T3; two hours after skin incision, T4; at the end of surgery. HR: heart rate, MAP: mean arterial pressure, CVP: central venous pressure, CI: cardiac index, SV: stroke volume, SVI: stroke volume index, $\mathrm{DO}_{2} \mathrm{I}$ : oxygen delivery index, $\mathrm{SCVO}_{2}$ : central venous oxygen saturation. ${ }^{\mathrm{a}} \mathrm{p}<0.05$; ${ }^{\mathrm{b}} \mathrm{p}<0.01$ relative to $\mathrm{T} 1$ within the same group. ${ }^{\mathrm{c}} \mathrm{p}<0.05 ;{ }^{\mathrm{d}} \mathrm{p}<0.01$; relative to HES group. 
Table 3: Fluid balance

\begin{tabular}{|l|c|c|}
\hline & HES group $(\mathrm{n}=25)$ & GEL group $(\mathrm{n}=25)$ \\
\hline Blood loss $(\mathrm{ml})$ & $1308.10 \pm 335.01$ & $1180.02 \pm 318.85$ \\
\hline Blood given $(\mathrm{ml})$ & $488.01 \pm 438.10$ & $320.30 \pm 244.95$ \\
\hline Crystalloid $(\mathrm{ml})$ & $5120.30 \pm 1542.99$ & $4500.04 \pm 1118.03$ \\
\hline Colloid $(\mathrm{ml})$ & $760.04 \pm 246.63$ & $624.02 \pm 296.20$ \\
\hline Plasma & $3(12 \%)$ & $0(0 \%)$ \\
\hline Platelets & $1(4 \%)$ & $0(0 \%)$ \\
\hline Urine output $(\mathrm{ml}$ at the end of surgery) & $896.03 \pm 117.19$ & $824.04 \pm 263.44$ \\
\hline
\end{tabular}

Data are expressed as mean \pm SD or number of patients (\%).

Table 4: Blood and coagulation tests

\begin{tabular}{|c|c|c|c|c|c|c|c|}
\hline & Groups & Pre-operative & Day 1 & Day 2 & Day 3 & Day 4 & Day 5 \\
\hline \multirow[t]{2}{*}{$\mathrm{Hb}(\mathrm{g} / \mathrm{dl})$} & HES group & $12.30 \pm 1.28$ & $\begin{array}{c}10.13 \pm \\
1.41^{\mathrm{b}}\end{array}$ & $\begin{array}{c}9.59 \pm \\
1.12^{\mathrm{b}}\end{array}$ & $\begin{array}{c}9.53 \pm \\
0.98^{\mathrm{b}}\end{array}$ & $\begin{array}{c}10.07 \pm \\
0.78^{\mathrm{b}}\end{array}$ & $\begin{array}{c}10.52 \pm \\
1.03^{\mathrm{b}}\end{array}$ \\
\hline & GEL group & $12.14 \pm 2.17$ & $\begin{array}{l}9.89 \pm \\
1.79^{\mathrm{b}}\end{array}$ & $\begin{array}{l}9.62 \pm \\
2.04^{\mathrm{b}}\end{array}$ & $\begin{array}{l}9.63 \pm \\
2.27^{\mathrm{b}}\end{array}$ & $\begin{array}{l}9.97 \pm \\
2.18^{\mathrm{b}}\end{array}$ & $\begin{array}{l}9.92 \pm \\
1.96^{\mathrm{b}}\end{array}$ \\
\hline \multirow{2}{*}{$\begin{array}{l}\text { Hematocrit } \\
(\%)\end{array}$} & HES group & $36.60 \pm 3.58$ & $\begin{array}{c}30.26 \pm \\
3.36^{\mathrm{b}}\end{array}$ & $\begin{array}{c}28.54 \pm \\
3.25^{\mathrm{b}}\end{array}$ & $\begin{array}{c}28.87 \pm \\
2.15^{\mathrm{b}}\end{array}$ & $\begin{array}{c}29.61 \pm \\
1.19^{\mathrm{b}} \\
\end{array}$ & $\begin{array}{c}31.20 \pm \\
1.62^{\mathrm{b}} \\
\end{array}$ \\
\hline & GEL group & $36.69 \pm 5.36$ & $\begin{array}{c}29.80 \pm \\
4.97^{\mathrm{b}} \\
\end{array}$ & $\begin{array}{c}28.64 \pm \\
5.46^{\mathrm{b}} \\
\end{array}$ & $\begin{array}{c}28.45 \pm \\
5.84^{\mathrm{b}}\end{array}$ & $\begin{array}{c}29.33^{ \pm} \\
6.26^{\mathrm{b}} \\
\end{array}$ & $\begin{array}{r}29.96 \\
\pm 4.59 \mathrm{bc}\end{array}$ \\
\hline \multirow{2}{*}{$\begin{array}{l}\text { Platelets } \\
\left(10^{9} / \mathrm{L}\right)\end{array}$} & HES group & $\begin{array}{c}235.88 \pm \\
116.72 \\
\end{array}$ & $\begin{array}{c}213.72 \pm \\
70.17 \\
\end{array}$ & $\begin{array}{c}203.64 \pm \\
57.50^{\mathrm{a}} \\
\end{array}$ & $\begin{array}{c}215.80 \pm \\
35.51 \\
\end{array}$ & $\begin{array}{c}205.12 \pm \\
85.99 \\
\end{array}$ & $\begin{array}{c}275.28 \pm \\
49.67 \\
\end{array}$ \\
\hline & GEL group & $211.44 \pm 50.63$ & $\begin{array}{l}158.80 \pm \\
45.33^{\mathrm{bd}}\end{array}$ & $\begin{array}{l}149.68 \pm \\
44.02^{\mathrm{bd}}\end{array}$ & $\begin{array}{l}151.36 \pm \\
39.02^{\mathrm{bd}}\end{array}$ & $\begin{array}{l}169.84 \pm \\
58.54^{\mathrm{bd}}\end{array}$ & $\begin{array}{l}184.16 \pm \\
59.75^{\text {ad }}\end{array}$ \\
\hline \multirow{2}{*}{ PT (sec) } & HES group & $12.61 \pm 1.25$ & $\begin{array}{c}16.90 \pm \\
3.34^{\mathrm{b}}\end{array}$ & $\begin{array}{c}17.01{ }^{ \pm} \\
3.04^{\mathrm{b}}\end{array}$ & $\begin{array}{c}16.27 \pm \\
3.19^{\mathrm{b}}\end{array}$ & $\begin{array}{c}14.38 \pm \\
2.26^{\mathrm{b}}\end{array}$ & $\begin{array}{c}14.23 \pm \\
3.07^{\mathrm{b}}\end{array}$ \\
\hline & GEL group & $12.68 \pm 0.75$ & $\begin{array}{c}15.95^{ \pm} \\
2.91^{\mathrm{b}}\end{array}$ & $\begin{array}{c}15.14^{ \pm} \\
1.41^{\mathrm{b}} \\
\end{array}$ & $\begin{array}{c}14.78 \pm \\
1.52^{\mathrm{b}} \\
\end{array}$ & $\begin{array}{c}13.43 \pm \\
1.55 \\
\end{array}$ & $\begin{array}{c}13.11 \pm \\
1.25\end{array}$ \\
\hline \multirow{2}{*}{ PC (\%) } & HES group & $86.83 \pm 14.22$ & $\begin{array}{l}57.60 \pm \\
17.31^{\mathrm{b}}\end{array}$ & $\begin{array}{l}56.00^{ \pm} \\
15.60^{\mathrm{b}}\end{array}$ & $\begin{array}{l}74.56 \pm \\
11.85^{\mathrm{b}}\end{array}$ & $\begin{array}{l}71.64^{ \pm} \\
16.74^{\mathrm{b}}\end{array}$ & $\begin{array}{l}74.51^{ \pm} \\
18.24^{\mathrm{b}}\end{array}$ \\
\hline & GEL group & $85.12 \pm 8.98$ & $\begin{array}{l}60.48 \pm \\
14.19^{\mathrm{b}}\end{array}$ & $\begin{array}{l}60.51 \pm \\
17.52^{\mathrm{b}}\end{array}$ & $\begin{array}{l}69.94 \pm \\
9.63^{\mathrm{b}} \\
\end{array}$ & $\begin{array}{c}78.52 \pm \\
12.03 \\
\end{array}$ & $\begin{array}{c}81.60 \pm \\
11.65 \\
\end{array}$ \\
\hline \multirow{2}{*}{ INR } & HES group & $1.09 \pm 0.10$ & $\begin{array}{c}1.45 \pm \\
0.3^{\mathrm{b}} \\
\end{array}$ & $\begin{array}{l}1.47 \pm \\
0.25^{\mathrm{b}}\end{array}$ & $\begin{array}{c}1.40 \pm \\
0.27 \\
\end{array}$ & $\begin{array}{l}1.24 \pm \\
0.19^{\mathrm{b}}\end{array}$ & $\begin{array}{l}1.23 \pm \\
0.27^{\mathrm{b}}\end{array}$ \\
\hline & GEL group & $1.09 \pm 0.06$ & $\begin{array}{l}1.38 \pm \\
0.26^{\mathrm{b}}\end{array}$ & $\begin{array}{l}1.37 \pm \\
0.15^{b}\end{array}$ & $\begin{array}{l}1.32 \pm \\
0.18^{\mathrm{b}}\end{array}$ & $\begin{array}{c}1.15 \pm \\
0.14 \\
\end{array}$ & $\begin{array}{c}1.12 \pm \\
0.11 \\
\end{array}$ \\
\hline
\end{tabular}

$\mathrm{Hb}$ : hemoglobin concentration, PT: prothrombin time, PC: prothrombin concentration, INR: international normalized ratio, ${ }^{\mathrm{a}} \mathrm{p}<0.05 ;{ }^{\mathrm{b}} \mathrm{p}<0.01$ relative to T1 within the same group. ${ }^{\mathrm{c}} \mathrm{p}<0.05 ;{ }^{\mathrm{d}} \mathrm{p}<0.01$; relative to HES group.

Table 5: kidney and liver function tests

\begin{tabular}{lccccccc}
\hline & Groups & Pre-operative & Day 1 & Day 2 & Day 3 & Day 4 & Day 5 \\
\hline Creatinine & HES & $1.44 \pm 0.98$ & $1.28 \pm 0.75$ & $1.28 \pm 0.80$ & $1.23 \pm 0.78^{\mathrm{a}}$ & $1.23 \pm 0.77^{\mathrm{a}}$ & $1.11 \pm 0.56^{\mathrm{a}}$ \\
(mg/dl) & GEL & $1.38 \pm 0.55$ & $1.30 \pm 0.38$ & $1.41 \pm 0.59^{\mathrm{c}}$ & $1.34 \pm 0.62$ & $1.15 \pm 0.51^{\mathrm{b}}$ & $1.17 \pm 0.42^{\mathrm{b}}$ \\
AST (IU) & HES & $27.16 \pm 8.65$ & $55.60 \pm 29.39^{\mathrm{b}}$ & $60.56 \pm 41.39^{\mathrm{b}}$ & $48.72 \pm 44.39^{\mathrm{a}}$ & $45.92 \pm 46.05$ & $39.52 \pm 34.16$ \\
& GEL & $23.60 \pm 7.49$ & $33.36 \pm 19.44^{\mathrm{bd}}$ & $34.48 \pm 17.23^{\mathrm{b}}$ & $29.24 \pm 14.26^{\mathrm{a}}$ & $29.76 \pm 16.78^{\mathrm{a}}$ & $28.40 \pm 18.11$ \\
ALT (IU) & HES & $35.48 \pm 8.85$ & $39.16 \pm 14.85$ & $39.68 \pm 11.09$ & $36.20 \pm 15.16$ & $36.28 \pm 15.48$ & $34.04 \pm 18.20$ \\
& GEL & $35.48 \pm 11.46$ & $45.40 \pm 26.31^{\mathrm{a}}$ & $56.44 \pm 44.67^{\mathrm{a}}$ & $38.24 \pm 18.51$ & $36.04 \pm 23.87$ & $37.32 \pm 29.27$ \\
Bilirubin & HES & $0.78 \pm 0.60$ & $1.36 \pm 1.03^{\mathrm{b}}$ & $1.18 \pm 1.25$ & $0.91 \pm 0.54$ & $0.82 \pm 0.50$ & $0.68 \pm 0.22$ \\
(mg/dl) & GEL & $0.58 \pm 0.19$ & $0.74 \pm 0.29$ & $0.82 \pm 0.29^{\mathrm{b}}$ & $0.78 \pm 0.27^{\mathrm{b}}$ & $0.66 \pm 0.24$ & $0.65 \pm 0.32$ \\
\hline
\end{tabular}

AST: aspartate aminotransferase, ALT: alanine aminotransferase. ${ }^{\mathrm{a}} \mathrm{p}<0.05 ;{ }^{\mathrm{b}} \mathrm{p}<0.01$ relative to T1 within the same group. ${ }^{\mathrm{c}} \mathrm{p}<$ $0.05 ;{ }^{\mathrm{d}} \mathrm{p}<0.01$; relative to HES group.

\section{Discussion}

The current study showed that optimization of intravascular volume and tissue perfusion was comparable for both HES and GEL groups when using esophageal Doppler monitoring and delivering a goal directed protocol during elective major abdominal surgeries. There was no significant difference between the volumes of both colloids given to achieve the required hemodynamic 
endpoints. However, there was a negative effect of GEL on platelet count compared to HES, but without significant difference as regards blood loss between both groups. Hemodynamic data (HR and MAP), were higher at different readings when compared to baseline in both groups. This may be explained by insufficient anesthetic depth level which was not monitored in this study. Central venous pressure (CVP) was higher in GEL group than the HES group which could be explained by the difference in the baseline readings between the two groups.

Doppler derived data (CI, SV, SVI, peak velocity, systolic flow time, $\mathrm{DO}_{2} \mathrm{I}$ and $\mathrm{SCVO}_{2}$ ), showed no significant difference between both groups, which renders the two replacement therapies equally efficient. The results of this study go in accordance with Van der Linden et al. (2005) who concluded that all hemodynamic variables (HR, MAP, CI, SVI, $\mathrm{DO}_{2} \mathrm{I}$ and $\mathrm{SVO}_{2}$ ) using pulmonary artery catheter were similar when using HES 130/0.4 and modified fluid gelatin up to $20 \mathrm{~h}$ postoperatively. Measurement of cardiac output using the low morbidity technique of esophageal Doppler monitoring has been shown to correlate well with invasive methods such as thermodilution (Singer and Bennett, 1991). The optimization of intravascular volume using esophageal Doppler in multiple-trauma patients is associated with a decrease of blood lactate levels, a lower incidence of infectious complications, and a reduced duration of ICU and hospital stays (Chytra et al, 2007).

According to Abbas and Hill (2008), goaldirected fluid therapy using TED provides immediate optimization of tissue perfusion without invasive monitoring. In a stratified meta-analysis, Corcoran et al. (2012) demonstrated that perioperative outcomes in major surgeries favored a GDT using TED targeted a validated and objectively measurable hemodynamic variable, such as $\mathrm{CO}$ and FTc rather than conventional measures such as arterial blood pressure, urine output, or central venous pressure.
This study revealed no significant difference between both groups as regards the blood loss, blood given, amount of crystalloid and colloids administered during the study. The maximal recommended daily dose of either colloid was not exceeded. The number of patients required plasma or platelet transfusion as well as urine output were comparable between both groups. These results go in accordance with Haisch et al, (2001) who found that the usage of these two types of colloid did not differ as regard the blood loss and the use of packed red blood cells and fresh frozen plasma in patients undergoing cardiac surgery. These results were not in agreement with Mortelmans et al. (1995) who found higher post operative blood loss with HES 130/0.4 than Gelatin. Also, the results showed that HES and GEL were associated with equivalent effects on blood coagulation and hemostasis (PT, PC and INR). This is in concordance with a cardiac surgery study by Van der Linden et al. (2005) who found that laboratory tests $(\mathrm{Hb}, \mathrm{Hct}, \mathrm{PT}, \mathrm{APPT})$ as well as measured blood loss and calculated net RBC loss were similar in both types of colloid. Neither the number of patients transfused nor the volume of blood products given differed. This was also confirmed by Casutt et al. (2010), who measured quality of clot formation using two viscoelastic coagulation tests; SONOCLOT ${ }^{\mathrm{TM}}$ and activated rotation thromboelasto-metry ROTEM ${ }^{\mathrm{TM}}$. Also, Schabinski et al. (2009) found that the HES 130/0.4 preparation showed similar changes in thromboelastographic data compared with a gelatin group in patients undergoing cardiac surgery with cardio pulmonary bypass.

In contrast, Inal et al. (2010) showed that global tests of coagulation (PT and aPTT) and platelet aggregation were only slightly influenced by Gelatin used in orthopedic patients undergoing acute normovolemic hemodilution when compared to patients received high molecular weight-HES preparation which differs from HES 130/0.4 (Voluven) used in this study. Shramko et al. 
(2010) were not in accordance with us as their study revealed that both HES 130/0.4 and Gelatin impair clot formation and firmness shortly after cardiac surgery in a dosedependent fashion by using ROTEM, but neither colloid increased blood loss.

As regards platelet count, this study showed significant decrease of platelet count in GEL group throughout the five postoperative days when compared to the baseline and also when compared to HES group which showed only significant reduction on $2^{\text {nd }}$ postoperative day. Van der Linden et al. (2005) found significant postoperative reduction of platelet counts with HES and GEL groups compared to baseline but without significant difference when comparing the two groups. It was previously suggested that modified fluid gelatin solutions might influence the weight and reticular network of fibrin strands and platelet function with decreased von Willebrand factor (Thaler et al, 2005). However, other studies have shown only a moderate effect (Huttner et al, 2000) or indicate that blood coagulation might even be accelerated (Innerhofer et al, 2002). HES compromised blood coagulation significantly in vitro (Kozek-Langenecker, 2005) and in vivo (Innerhofer et al, 2002). There are several mechanisms which influence blood coagulation, for example, an acquired von Willebrand syndrome, reducing effect on factor VIII or decreased expression and activation of platelet surface GPIIb-IIIa receptor with impaired platelet adhesion and aggregation (Kozek-Langenecker, 2005).

Creatinine level revealed postoperative reduction in both groups compared to baseline being significantly lower on $2^{\text {nd }}$ postoperative day in group HES than group GEL. Mahmood et al. (2007) demonstrated that HES 130/0.4 maintained glomerular and tubular functions throughout the postoperative period more than Gelatin in patients undergoing abdominal aortic aneurysm surgery. They used more sensitive biochemical markers for renal functions which are able to detect early changes in renal functions. An- other study done by Boldt et al. (2008) on cardiac patients over 80 years, found that volume replacement with HES 130/0.4 was associated with less marked changes in kidney functions and endothelial inflammatory response than Gelatin.

This study showed elevation of liver functions (AST, ALT and total bilirubin), at different timings compared to baseline in each group which may be due to the effects of prolonged duration of surgery and anesthesia. They were comparable between both groups except in the first postoperative day where AST level was significantly higher in group HES than group GEL. In contrast, Van der Linden et al. (2005) found that no significant difference in creatinine, AST and ALT between these two types of colloid. Also, Inal et al. (2010) found that no significant change in liver functions measured by Indocyanine green plasma disappearance elimination values after administration of HES 130/0.4. The difference in results may be attributed to the different liver function test used in this study. A limitation of the current study would be assessing hemostasis using the routine screening laboratory tests, and recommendation of using the more accurate and specific hemostatic markers as sP-selectin, D-dimer and Von Willebrand factor to ascertain and compare impact of different colloids on hemostasis are required.

\section{Conclusion}

Intraoperative use of HES 130/0.4 was as effective as modified fluid gelatin in intravascular volume expansion guided by transesophageal Doppler. Administration of the HES 130/0.4 has a more favorable effect on platelet counts than Gelatin in major abdominal surgery but longer follow up is recommended. Future studies encompassing the integrity of TED as a monitor for optimization of fluid replacement and tissue perfusion are also recommended. Other studies to compare and ascertain the benefits of the different modalities of GDT using different colloids are required. 


\section{References}

Abbas, SM, Hill, AG, 2008: Systematic review of the literature for the use of oesophageal Doppler monitor for fluid replacement in major abdominal surgery. Anesthesia 63:44-51.

Boldt, J, Brosch, Ch, Röhm, K, Papsdorf, M, Mengistu, A, 2008: Comparison of the effects of gelatin and a modern hydroxyethyl starch solution on renal function and inflammatory response in elderly cardiac surgery patients. Br. J. Anaesth. 100:457-64.

Casutt, M, Kristoffy, A, Schuepfer, G, Spahn, DR, Konrad, C, 2010: Effects on coagulation of balanced (130/0.42) and non-balanced (130/0.4) hydroxyethyl starch or gelatin compared with balanced Ringer's solution: an in vitro study using two different viscoelastic coagulation tests ROTEM $^{\mathrm{TM}}$ and SONOCLOT ${ }^{\mathrm{TM}}$. Br. J. Anesth. 105:273-81.

Chappell, D, Jacob, M, Hofmann-Kiefer, K, Conzen, P, Rehm, M, 2008: A rational approach to perioperative fluid management. Anesthesiology 109:723-40.

Chytra, I., Pradl, R., Bosman, R., Pelnár, P., Kasal, E., Zidková, A. 2007: Esophageal Doppler-guided fluid management decreases blood lactate levels in multiple-trauma patients: a randomized controlled trial. Crit. Care 11: R24.

Corcoran, T., Rhodes, J.E., Clarke, S., Myles, P.S., Ho, K.M. 2012: Perioperative fluid management strategies in major surgery: a stratified meta-analysis. Anesth. Analg. 114: 640-51.

Dark, PM, Singer, M, 2004: The validity of trans-esophageal Doppler ultrasono-graphy as a measure of cardiac output in critically ill adults. Intensive Care Med. 30: 2060-6.

Franz, A, Bräunlich, P, Gamsjäger, T, Felfernig, M, Gustorff, B, et al, 2001: The effects of hydroxyethyl starches of varying molecular weights on platelet function. Anesth. Analg. 92:1402-7.

Gan, TJ, Soppitt, A, Maroof, M, el-Moalem, H, Robertson, KM, et al, 2002: Goal-directed intraoperative fluid administration reduces length of hospital stay after major surgery. Anesthesiology 97:820-6.

Gattas, DJ, Dan, A, Myburgh, J, Billot, L, Lo, S, et al, 2012: Fluid resuscitation with $6 \%$ hydroxyethyl starch $(130 / 0.4)$ in acutely ill patients: an updated systematic review and metaanalysis. Anesth. Analg. 114:159-69.
Haisch, G, Boldt, J, Krebs, C, Kumle, B, Suttner, S, et al, 2001: The influence of intravascular volume therapy with a new hydroxyethyl starch preparation (6\% HES 130/0.4) on coagulation in patients undergoing major abdominal surgery. Anesth. Analg. 92: 565-71.

Huttner, I., Boldt, J, Haisch, G, Suttner, S, Kumle, B, et al, 2000: Influence of different colloids on molecular markers of haemostasis and platelet function in patients undergoing major abdominal surgery. Br. J. Anaesth. 85:41723.

Inal, MT, Memiş, D, Karamanlioglu, B, Sut, N, 2010: Effects of polygeline and hydroxyethyl starch solutions on liver functions assessed with LIMON in hypovolemic patients. J. Crit. Care 25:361-5.

Innerhofer, P, Fries, D, Margreiter, J, Klingler, A, Kühbacher, G, et al, 2002: The effects of perioperatively administered colloids and crystalloids on primary platelet mediated hemostasis and clot formation. Anesth. Analg. 95:858-65.

Jin, SL, Yu, BW, 2010: Effects of acute hypervolemic fluid infusion of hydroxyethyl starch and gelatin on hemostasis and possible mechanisms. Clin. Appl. Thromb. Hemost. 16:91-8.

Jungheinrich, C, Sauermann, W, Bepperling, F, Vogt, NH, 2004: Volume efficacy and reduced influence on measures of coagulation using hydroxyethyl starch 130/0.4 (6\%) with an optimised in vivo molecular weight in orthopaedic surgery: a randomised, double-blind study. Drugs R D 5:1-9.

Kim, JY, Lee, JW, Kweon, TD, Kwak, YL, Kim, JH, et al, 2007: The effect of 6\% hydroxyethyl starch 130/0.4 on hemostasis and hemodynamic efficacy in off-pump coronary artery bypass surgery: a comparison with $6 \%$ hydroxyethyl starch 200/0.5. Korean J. Anesthesiol. 53:S14-21.

Kozek-Langenecker, SA, 2005: Effects of hydroxyethyl starch solutions on hemostasis. Anesthesiology 103:654-60.

Mahmood, A, Gosling, P, Vohra, RK, 2007: Randomized clinical trial comparing the effects on renal function of hydroxyethyl starch or gelatine during aortic aneurysm surgery. Br. J. Surg. 94:427-33.

Mastroianni, L, Low, HB, Rollman, J, Wagle, M, Bleske, B, et al, 1994: A comparison of $10 \%$ pentastarch and 5\% albumin in patients undergo- 
ing open-heart surgery. J. Clin. Pharmacol. 34:34-40.

Mortelmans, YJ, Vermaut, G, Verbruggen, AM, Arnout, JM, Vermylen, J, et al, 1995: Effects of 6\% hydroxyethyl starch and 3\% modified fluid gelatin on intravascular volume and coagulation during intraoperative hemodilution. Anesth. Analg. 81:1235-42.

Mythen, MG, Webb, AR, 1994: Intraoperative gut mucosal hypoperfusion is associated with increased post-operative complications and cost. Intensive Care Med. 20:99-104.

Schabinski, F, Oishi, J, Tuche, F, Luy, A, Sakr, Y, et al. 2009: Effects of a predominantly hydroxyethyl starch (HES)-based and a predominantly non HES-based fluid therapy on renal function in surgical ICU patients. Intensive Care Med. 35: 1539-47.

Schramko, A, Suojaranta-Ylinen, R, Kuitunen, A, Raivio, P, Kukkonen, S, et al, 2010: Hydroxyethylstarch and gelatin solutions impair blood coagulation after cardiac surgery: a prospective randomized trial. Br. J. Anaesth. 104: 691-7.
Singer, M, Bennett, ED, 1991: Noninvasive optimization of left ventricular filling using oesophageal Doppler. Critical Care Med. 19:11327.

Thaler, U, Deusch, E, Kozek-Langenecker, SA, 2005: In vitro effects of gelatin solutions on platelet function: a comparison with hydroxyethyl starch solutions. Anesthesia 60:554-9.

Treib, J, Baron, JF, Grauer, MT, Strauss, RG, 1999: An international view of hydroxyethyl starches. Intensive Care Med. 25:258-68.

Van Der Linden, PJ, Schmartz, D, 1992: Pharmacology of gelatins. In: Baron JF, ed. volume expansion. Paris: Arnette 67-74.

Van der Linden, PJ, De Hert, SG, Deraedt, D, Cromheecke, S, De Decker, K, et al, 2005: Hydroxyethyl starch 130/0.4 versus modified fluid gelatin for volume expansion in cardiac surgery patients: the effects on perioperative bleeding and transfusion needs. Anesth. Analg. 101:629-34.

Van Der Linden, PJ, James, M, Mythen, M, Weiskopf, RB, 2013: Safety of modern starches used during surgery. Anesth. Analg. 116:35-48. 